\title{
MATCHING THEOREMS ON TOPOLOGICAL SPACES
}

\author{
Ivan D. Aranđelović
}

\begin{abstract}
In this paper we give one general matching theorem and some its applications in the fixed point theory. Our results generalize earlier theorems obtained by Horvath, Chang - Zhang and Chang - Ma.
\end{abstract}

\section{Introduction}

The famous Brouwer fixed point theorem (any continuous function $f: K \rightarrow K$ has at least one fixed point, where $K \subseteq R^{n}$ is non-empty, compact and convex set of $R^{n}$ ) for $n=3$ was proved by him in 1909; equivalent results was established earlier by Henri Poincare in 1883 and P. Bohl in 1904. It was Hadamard who in 1910 gave (using the Kronecker index) the first proof for an arbitrary $n$. In 1912 Brouwer gave another proof using the simplicial approximation technique, and notions of degree. A short and simple proof of Brouwer theorem was given in 1929 by Knaster, Kuratowski and Mazurkiewicz. This proof is based on the following corollary of Sperner's lemma:

Proposition A Let $F_{0}, \ldots, F_{n}$ be nonempty subsets of $R^{n+1}$, such that $\Delta_{A} \subseteq \bigcup_{e_{j} \in A} F_{j}$, where $A \subseteq\left\{e_{0}, \ldots, e_{n}\right\}$. If all $F_{j}, 0 \leq j \leq n$, are closed sets then

$$
\bigcap_{0 \leq i \leq n} F_{i} \neq \emptyset
$$

This result is known as the KKM lemma. There are many its applications and generalizations in Functional Analysis and Topology (see [8]).

It's interesting that this statement is true, when all $F_{j}, 0 \leq j \leq n$, are open sets. This result is due to Shih [9] and Kim [7], sixty years ago.

AMS (MOS) Subject Classification 1991. Primary: 54E15, 54D30, 54H25.

Key words and phrases: Matching theorem, fixed point. 


\section{Preliminaries}

Let $X$ and $Y$ be nonempty sets; we denote by $2^{X}$ a family of all nonempty subsets of $X, \mathcal{F}(X)$ a family of all nonempty finite subsets of $X$ and $\mathcal{P}(X)$ a family of all subsets of $X$. A multifunction $G$ from $X$ into $Y$ is a map $G: X \rightarrow 2^{Y}$. If $G: X \longrightarrow 2^{Y}$ we define $G^{-1}, G^{*}: Y \longrightarrow \mathcal{P}(X)$ and $G^{c}$ : $X \longrightarrow \mathcal{P}(Y)$ by $G^{-1}(y)=\{x \in X: y \in G(x)\}, G^{*}(y)=\{x \in X: y \notin G(x)\}$ and $G^{c}(x)=Y \backslash G(x)$. Clearly, by definitions we have $\left(G^{c}\right)^{c}=G$ and for all $x \in X, y \in Y$ statements $y \in G(x)$ and $x \in G^{-1}(y)$ are equivalent which implies $\left(G^{-1}\right)^{-1}=G$.

Let $X$ be a nonempty set and let $F: X \longrightarrow \mathcal{P}(X)$ be a multifunction. $x_{0} \in X$ is a fixed point of the multifunction $F$ if and only if $x_{0} \in F\left(x_{0}\right)$. Since statements $x \in F(x)$ and $x \in F^{-1}(x)$ are equivalent, multifunctions $F$ and $F^{-1}$ have the same fixed points.

The following notations are used: $\left[e_{0}, \ldots, e_{n}\right]$ for the canonical basis of $R^{n+1}, \Delta_{n}=\operatorname{co}\left(\left[e_{0}, \ldots, e_{n}\right]\right)$ for the standard simplex of dimension $n$, where $c o($.$) denotes the convex hull. \Delta_{A}=c o A$, where $A \subseteq\left\{e_{0}, \ldots, e_{n}\right\} . \Delta_{n}^{k}$ is the $k$ - skeleton of $\Delta_{n}$ i.e. it is the union of all $k$-dimensional faces of $\Delta_{n}$. If $X$ is a path-connected topological space, $\mathcal{H}_{n}(X)$ is the homotopy group of order $n$, where $n \in N$.

Definition 2 (Aranđelović [1]) Let $X$ be a uniform space. Measure of noncompactness on $X$ is an arbitrary function $\phi: \mathcal{P}(X) \rightarrow[0, \infty]$, which satisfies the following conditions:

1) $\Phi(A)=\infty$ if and only if set $A$ is unbounded;

2) $\Phi(A)=\phi(\bar{A})$;

3) from $\Phi(A)=0$ it follows that $A$ is totally bounded set;

4) from $A \subseteq B$ it follows $\Phi(A) \leq \Phi(B)$;

5) if $X$ is complete space, and if $\left\{B_{n}\right\}_{n \in N}$ is a sequence of closed subsets of $X$ such that $B_{n+1} \subseteq B_{n}$ for each $n \in N \lim _{n \rightarrow \infty} \Phi\left(B_{n}\right)=0$, then $K=\bigcap_{n \in N} B_{n}$ is nonempty compact set.

A family of sets has the finite intersection property if and only if the intersection of each its finite subfamily is nonempty.

Proposition B (Aranđelović [1]) Let $X$ be a complete uniform space, $\Phi$ measure of noncompactness on $X$ and $\left\{G_{j} \mid j \in J\right\} \subseteq \mathcal{P}(\mathcal{X})$ a family of its closed subsets which has finite intersection property such that for all $t>0$ there exists finite set $A \subseteq J$ such that $\Phi\left(\bigcap_{j \in A} G_{j}\right)<t$. Then

$$
\bigcap_{j \in J} G_{j} \neq \emptyset \text {. }
$$

For a nonempty set $X$ by $\Delta_{X}=\{(x, x) \mid x \in X\}$ we denote its diagonal. 


\section{Main Results}

Theorem 1. Let $X$ be a topological space, $I$ a nonempty set and $H$ : $\mathcal{F}(I) \longrightarrow 2^{X}$ is a given multifunction which satisfies the following condition:

1) if $\{x\} \subseteq I$ then $H(\{x\}) \neq \emptyset$;

2) if $A \in \mathcal{F}(I)$ then $H(A)$ is a path-connected set;

3) if $A \in \mathcal{F}(I)$ has a $n \geq 3$ members, then $\mathcal{H}_{n-2}(H(A))$ is a trivial group;

4) if $A, B \in \mathcal{F}(I)$ and $A \subseteq B$ then $H(A) \subseteq H(B)$.

If $A=\left\{x_{0}, \ldots, x_{n}\right\} \in \mathcal{F}(I)$, then there exist a continuous map $f$ : $\Delta_{n} \rightarrow X$ such that

$$
f\left(\Delta_{B}\right) \subseteq H\left(\left\{x_{j}: j \in B\right\}\right)
$$

for each $B \subseteq\{0, \ldots, n\}$.

Proof We use the following statement: let $X$ be a topological space such that $\mathcal{H}_{n-2}(X)$ (for some $n \geq 3$ ) is a trivial group; then any continuous function is the restriction to $\partial \Delta_{n}$ (the boundary of $\Delta_{n}$ ) of a continuous function $g$ : $\Delta_{n} \rightarrow X$ (see Spanier [10]). Let $y_{0}, \ldots, y_{n} \in X$ be arbitrary elements such that $y_{k} \in H\left(\left\{x_{k}\right\}\right)$ for any $0 \leq k \leq n$. Function $f_{0}:\left\{e_{0}, \ldots, e_{n}\right\} \rightarrow X$ defined by $f_{0}\left(e_{j}\right)=y_{j}$ is continuous because its domain is a finite subset of an Euclidian space. The sets $H\left(\left\{y_{i}, y_{j}\right\}\right), i \neq j, i \neq j$ are path-connected. Every path which connects points $y_{i}$ i $y_{j}, 0 \leq i<j \leq n$, defines a continuous function on the interval $[0,1]$ into $X$. So, we can define a continuous function $f_{1}$ : $\Delta_{n}^{1} \rightarrow X$, such that $\left.f_{1}\right|_{\Delta_{n}^{0}}=f_{0}$, because $[0,1]$ and $\left[e_{i}, e_{j}\right]$ are homeomorphic sets. This function, also satisfies condition $f\left(\left[e_{i}, e_{j}\right]\right) \subseteq H\left(\left\{y_{i}, y_{j}\right\}\right), i \neq$ $j, i \neq j$ for any $1 \leq i<j \leq n$. Let $1<k<n$ and let $f_{k}: \Delta_{n}^{k} \rightarrow X$ be a continuous function, which satisfies the following condition: for any finite set $J \subseteq\{0, \ldots, n\}$, which has $k$ elements, $f_{k}\left(\Delta_{J}\right) \subseteq H\left(\left\{x_{j} \mid j \in J\right\}\right)$. If $J^{\prime} \subseteq\{0, \ldots, n\}$ has $k+1$ elements then $\partial \Delta_{J^{\prime}} \subseteq \Delta_{n}^{k}$. Since $f_{k}\left(\partial \Delta_{J^{\prime}}\right) \subseteq$ $H\left(\left\{\left(x_{j} \mid j \in J^{\prime}\right\}\right)\right.$ and $\mathcal{H}_{k-1}\left(H\left(\left\{\left(x_{j} \mid j \in J^{\prime}\right\}\right)\right)\right.$ is a trivial group then there exists a continuous mapping $f_{k+1, J}: \Delta_{J^{\prime}} \rightarrow X$ such that $\left.f_{k+1, J^{\prime}}\right|_{\partial \Delta_{J^{\prime}}}=f_{k}$. So we have an extension $f_{k+1}$ of $f_{k}$ on $\Delta_{n}^{k+1}$, because if $\Delta_{J 1}$ and $\Delta_{J 2}$ are two $k+1$-dimensional faces with the nonempty intersection; then $\Delta_{J 1} \bigcap \Delta_{J 2} \subseteq \Delta_{n}^{k}$ and $f_{k+1, J 1}(x)=f_{k+1, J 2}(x)=f_{k}(x)$ for any $x \in \Delta_{J 1} \cap \Delta_{J 2} \subseteq \Delta_{n}^{k}$. The continuous function $f^{n}=f: \Delta_{n} \rightarrow X$ has the following property: $f\left(\Delta_{J}\right) \subseteq H\left(\left\{\left(x_{j} \mid j \in J\right\}\right)\right.$, for any $J \subseteq\{0, \ldots, n\}$.

If $H(A)$ is contractible set for any $A \in \mathcal{F}(X)$ from Theorem 1 we obtain a famous result of Horvath (see [4] and [6]). 
In the next theorem we give sufficiency and neccesity conditions for finite intersection property for arbitrary family of subsets of arbitrary topological space. First of them generalize earlier results of Chang and Zhang [3] and Chang and Ma [2].

Theorem 2. Let $X$ be a topological space and I a nonempty set. Family of closed [open] subsets of space $X\left\{G_{i}\right\}_{i \in I}$ has the finite intersection property if and only if for any $J \in \mathcal{F}(I)$ there exists mapping $H_{J}: 2^{J} \rightarrow 2^{X}$ which satisfies:

1) for each $A \in \mathcal{F}(J)$ set $H_{J}(A)$ is nonempty and path-connected;

2) if $A \in \mathcal{F}(I)$ has a $n \geq 3$ members, then $\mathcal{H}_{n-2}\left(H_{J}(A)\right)$ is a trivial group;

3) if $A, B \in \mathcal{F}(I)$ and $A \subseteq B$ then $H_{J}(A) \subseteq H_{J}(B)$;

4) from $L \in \mathcal{F}(J)$ it follows $H_{J}(L) \subseteq \bigcup_{j \in L} G_{j}$.

Proof Neccesity. If $\left\{G_{i} \mid i \in I\right\}$ has the finite intersection property, then any finite set $J \in 2^{I}$ satisfies $\bigcap_{j \in J} G_{j} \neq \emptyset$. For arbitrary $x_{*} \in \bigcap_{i=1}^{n} G\left(x_{i}\right)$ let $H_{J}(A)=\left\{x_{*}\right\}$ for $A \subseteq J$. This mapping satisfies conditions 1),2), 3) and 4).

Sufficiency. Let $\left\{G_{i}\right\}_{i \in I}$ satisfies conditions of the Theorem. If this family does not have finite intersection property, then there exists $J \in \mathcal{F}(I)$ such that:

$$
\bigcap_{j \in J} G_{j}=\emptyset
$$

If $J=\left\{y_{1}, y_{2}, \ldots, y_{n}\right\}$ from Theorem 1 it follows that there exists a continuous function $f: \Delta_{n} \rightarrow X$ such that $B \subseteq\{0, \ldots, n\}$ implies

$$
f\left(\Delta_{B}\right) \subseteq H_{J}\left(\left\{y_{j}: j \in B\right\}\right) .
$$

Let $\left\{G_{i}\right\}_{i \in I}$ be a family of closed [open] subsets. Then the family of closed [open] sets $\left\{G_{j}^{*} \mid j \in J\right\}$ defined by $G_{j}^{*}=f^{-1}\left(G_{j}\right)$ satisfies conditions of KKM lemma [Kim-Shih result] which implies $\bigcap_{j \in J} G_{j}^{*} \neq \emptyset$. So, there exists $x_{*} \in \bigcap_{j \in J} G_{j}^{*}$. Now $f\left(x_{*}\right) \in G_{j}$ for $j \in J$ which is a contradiction.

Next statements extend results of C. Horvath [4] and [6].

Corollary 1. Let $X$ be a complete uniform space, $\psi$ arbitrary measure of noncompactness defined on $X$ and $\left\{G_{j}\right\}_{j \in I}$ a family of closed subsets of $X$. If for any $t>0$ there exists a finite set $A \subseteq J$ such that $\psi\left(\bigcap_{x \in A} G_{j}\right)<t$ and if for any $J \in \mathcal{F}(I)$ there exist $H_{J}: 2^{J} \rightarrow 2^{X}$ such that:

1) for each $A \in \mathcal{F}(J)$ set $H_{J}(A)$ is nonempty and path-connected;

2) if $A \in \mathcal{F}(I)$ has a $n \geq 3$ members, then $\mathcal{H}_{n-2}\left(H_{J}(A)\right)$ is a trivial group;

3) if $A, B \in \mathcal{F}(I)$ and $A \subseteq B$ then $H_{J}(A) \subseteq H_{J}(B)$;

4) from $L \in \mathcal{F}(J)$ it follows $H_{J}(L) \subseteq \bigcup_{j \in L} G_{j}$. then $\bigcap_{j \in I} G_{j} \neq \emptyset$. 
Proof From Theorem 2 it follows that this family has the finite intersection property. From Proposition B it follows that $\bigcap_{j \in I} G_{j} \neq \emptyset$ because $X$ is a complete space.

\section{Applications in the Fixed point theory}

Now we give some applications of main results in fixed point theory, which extends earlier results of C. Horvath [5].

Theorem 3. Let $X$ be a compact path-connected topological space such that $\mathcal{H}_{n}(X)$ is a trivial group, for any $n \in \mathcal{N}$ and let $R: X \rightarrow \mathcal{P}(X)$ be an multifunction which satisfies:

1) $R^{-1} x$ is an open set for any $x \in X$;

2) $\bigcap_{x \in A} R(x) \neq \emptyset$ implies that $\mathcal{H}_{n}\left(\bigcap_{x \in A} R(x)\right)$ is a trivial group for any $A \in 2^{X}$ and $n \in \mathcal{N}$;

then either exists $x_{0} \in X$ such that $x_{0} \in R\left(x_{0}\right)$ or there exists $y_{0} \in X$ such that $R\left(y_{0}\right)=\emptyset$.

Proof Completeness and uniformity of the space $X$ follows from its compactness. For any $A \in \mathcal{F}(X)$ denote by $\tilde{A}$ the set $\{x \in X \mid A \subseteq R(x)\}$.

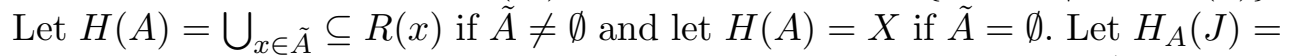
$H(J)$ for $J \in F(A)$. Let $A \in \mathcal{F}(X)$ be such that $H(A) \nsubseteq \bigcup_{x \in A} R^{*-1}(x)$. Then there exists $x_{0} \in H(A)$ such that $A \subseteq R\left(x_{0}\right)$. Now we have $H(A) \subseteq R\left(x_{0}\right)$ which implies $x_{0} \in R\left(x_{0}\right)$. In complementary case $H(A) \subseteq \bigcup_{x \in A} R^{*-1}(x)$, which implies by Theorem 2 that the family $\left\{R^{*-1}(x)\right\}_{x \in X}$ of closed sets has the finite intersection property. Compactness of $X$ implies $\bigcap_{x \in X} R^{*-1}(x) \neq \emptyset$. Hence there exists $y_{0} \in \bigcap_{x \in X} R^{*-1}(x)$ which implies $x \notin R\left(y_{0}\right)$ for each $x \in X$. So $R\left(y_{0}\right)=\emptyset$.

Theorem 4. Let $X$ be a compact path-connected topological space such that $\mathcal{H}_{n}(X)$ is a trivial group, for any $n \in \mathcal{N}$ and let $R: X \rightarrow 2^{X}$ be a multifunction which satisfies:

1) $R^{-1} x$ is an open set for each $x \in X$;

2) for each $A \in 2^{X}$ from $\bigcap_{x \in A} R(x) \neq \emptyset$ it follows that $\mathcal{H}_{n}\left(\bigcap_{x \in A} R(x)\right)$ is a trivial group, for any $n \in \mathcal{N}$.

Then for any continuous function $f: X \rightarrow X$ there exists $x_{0} \in X$ such that $x_{0} \in R\left(f\left(x_{0}\right)\right)$.

Proof The mapping $V$ defined by $V(x)=R(f(x))$ satisfies conditions of Theorem 3 and its values are nonempty sets. This implies that there exists $x_{0} \in X$ such that $x_{0} \in V\left(x_{0}\right)$. 
Theorem 5. Let $X$ be a compact path-connected Hausdorff topological space such that $\mathcal{H}_{n}(X)$ is a trivial group, for any $n \in \mathcal{N}$, and let $\left\{v_{i}\right\}_{i \in I}$ be a symmetric base (from $(x, y) \in v_{i}$ follows $(y, x) \in v_{i}$ ) of open (in topology of space $X \times X)$ neighborhoods of $\Delta_{X}$. Let $V_{i}(x)$ be $\left\{y \in X \mid(x, y) \in v_{i}\right\}$. If for any $A \in 2^{X}$ from $\bigcap_{x \in A} V_{i}(x) \neq \emptyset$ it follows that $\mathcal{H}_{n}\left(\bigcap_{x \in A} V_{i}(x)\right)$ is a trivial group for any $n \in \mathcal{N}$, then any continuous function $f: X \rightarrow X$ has at least one fixed point.

Proof If $f: X \rightarrow X$ is a continuous function without fixed points then

$$
\{(x, f(x)) \mid x \in X\} \bigcap \Delta=\emptyset .
$$

So then there exists $i_{0} \in I$ such that

$$
\{(x, f(x)) \mid x \in X\} \bigcap v_{i_{0}}=\emptyset .
$$

Multifunction $R: x \rightarrow V_{i_{0}}(x)$ satisfies conditions of Theorem 4 which implies that $x_{0} \in R\left(f\left(x_{0}\right)\right)$ for some $x_{0} \in X$ which is a contradiction.

This theorems when $X$ is contractible space reduce to Horvath results [5]. Its corollary is the next statement which is a generalization of famous results of Brouwer, Schauder, Tychonoff, Ky Fan and many others.

Corollary 2. Let $X$ be a Hausdorff topological commutative group, $K \subseteq X$ a compact path-connected set such that $\mathcal{H}_{n}(K)$ is a trivial group, for any $n \in \mathcal{N}$, and let $\left\{v_{i}\right\}_{i \in I}$ be a symmetric base (from $x \in V_{i}$ it follows $-x \in V_{i}$ ) of open neighborhoods of zero in $X$. If for any $A \in 2^{K}$ from $K \bigcap\left(\bigcap_{x \in A}\left(x+V_{i}\right)\right) \neq \emptyset$ it follows that $\mathcal{H}_{n}\left(K \bigcap\left(\bigcap_{x \in A} x+V_{i}\right)\right)$ is a trivial group for any $n \in \mathcal{N}$ then any continuous function $f: X \rightarrow X$ has at least one fixed point.

\section{References}

[1] Ivan Aranđelović: Measure of noncompactness on uniform spaces, Math. Moravica 2 (1998) 1-8.

[2] Shih-sen Chang and Yi-Hai Ma: Generalized KKM theorem on H-space with applications, J. Math. Anal. Appl. 163(1992), no. 2, 406-421.

[3] Sih-sen Chang and Ying Zhang: Generalized KKM theorem and variational Inequalities, J. Math. Anal. Appl. 159 (1991) 208-223.

[4] Charles Horvath: Point fixes et coincidences pour les applications multivoques sans convexite, C. R. Acad. Sc. Paris, 296, Serie I(1983) 403-406.

[5] Charles Horvath: Points fixes et coincidences dans les espaces topologiques compacts conctactiles, C.R.Acad.Sc. Paris, t.299, Serie II, 11(1984) 519-521. 
[6] Charles Horvath: Contractibility and generalized convexity, J. Math. Anal. Appl. 156 (1991), 341-357.

[7] Wan Kyu Kim: Some applications of Kakutani fixed point theorem, J. Math. Anal. Appl. 121 (1987), 119-122.

[8] Marc Lassonde: On use of KKM-multifunctions in fixed point theory and related topics, J. Math. Anal. and Appl. 97 (1983),151-201.

[9] Mau-Hsiang Shih: Covering properties of convex sets, Bull. London Math. Soc. 18 (1986) 57-59.

[10] Edwin Spanier: Algebraic topology, Mc Graw-Hill, New York 1966.

Faculty of Mechanical Engineering, 27 marta 80, 11000 Beograd. e-mail: iva@alfa.mas.bg.ac.yu 